\title{
Clinical Study \\ The Effect of Metabolic Syndrome upon the Success of Varicocelectomy
}

\author{
Ufuk Ozturk, ${ }^{1}$ Nevzat Can Sener, ${ }^{1}$ Ismail Nalbant, ${ }^{2}$ Osman Raif Karabacak, ${ }^{1}$ \\ Mustafa Gurhan Ulusoy, ${ }^{3}$ and M. Abdurrahim Imamoglu ${ }^{1}$ \\ ${ }^{1}$ Department of Urology, Ankara Dışkapı Yıldırım Beyazit Education and Research Hospital, Ministry of Health, \\ Ankara 06110, Turkey \\ ${ }^{2}$ Department of Urology, Yenimahalle State Hospital, Ministry of Health, Ankara, Turkey \\ ${ }^{3}$ Ankara Training and Research Hospital, Ministry of Health, Ankara, Turkey \\ Correspondence should be addressed to Ufuk Ozturk, ufukozturk71@gmail.com
}

Received 23 September 2012; Accepted 10 December 2012

Academic Editors: E. Baldi, O. W. Hakenberg, A. Komiya, and W. J. Oosterlinck

Copyright $($ C 2012 Ufuk Ozturk et al. This is an open access article distributed under the Creative Commons Attribution License, which permits unrestricted use, distribution, and reproduction in any medium, provided the original work is properly cited.

\begin{abstract}
We aimed to investigate the impact of metabolic syndrome (MetS) on the varicocele treatment. 101 patients underwent spermatic vein ligation between 2007 and 2010 were retrospectively analyzed. Those patients were divided into two groups as without ( $n: 56$, Group 1) or with MetS ( $n: 48$, Group 2). All the patients underwent left microsurgical subinguinal spermatic vein ligation. Groups were compared by the improvement on sperm parameters and spontaneous pregnancy rates at a mean of $19( \pm 4)$ months followup. When sperm parameters were compared postoperatively, the significant improvement in total sperm count, motile sperm count percentage, and normal sperm percentage was reported. The groups were compared to each other and the improvement seemed significantly better in Group 1. There was no statistically significant improvement difference in the normal sperm percentage between groups. Spontaneous pregnancy rate after two years was $45 \%$ in Group 1 and $34 \%$ in Group $2(P<0.05)$. Patients with MetS and varicocele improved after surgery, but not as well as the similar group without MetS. This may help to show that MetS can be a factor for male infertility.
\end{abstract}

\section{Introduction}

Metabolic syndrome (MetS) is an important problem in developed countries. In recent years, the prevalence of MetS is growing. The criteria that define MetS can be listed as having hypertension, high low-density lipoprotein (LDL) cholesterolemia, low high-density lipoprotein (HDL) cholesterolemia, hyperglycemia, obesity, physical inactivity, and blood coagulation disorder [1].

MetS can create or aggravate urologic diseases. Its associations with kidney stones [2], benign prostatic hyperplasia [3], and erectile dysfunction [4] are well defined. The association with an important andrologic issue, infertility, is extensively investigated recently $[5,6]$. However, it requires further and deeper investigation.

A treatable disease, varicocele, seems to be altered by MetS. We aimed to investigate the impact of MetS on varicocele treatment.

\section{Patients and Methods}

2.1. Patients. Patients undergone spermatic vein ligation between 2007 and 2010 were retrospectively analyzed with the local ethics committee approval. Those patients were divided into two groups as without ( $n$ : 56, Group 1) or with MetS ( $n$ : 48, Group 2). Color Doppler scrotal ultrasound was performed on all patients. Patients with varicocele were graded according to Dubin and Amelar clinical classification (Grade I: inducible during a Valsalva maneuver; Grade II: palpable; Grade III: visible) system [7]. Color Doppler scrotal ultrasound was performed with 5 to $10 \mathrm{MHz}$ probes during spontaneous breathing and under Valsalva maneuver. The Hirsh testicular Doppler classification (Grade I: no spontaneous venous reflux but inducible reflux with Valsalva maneuver; G II: intermittent spontaneous venous reflux: G III: continuous spontaneous venous reflux) was applied [8]. Group 1 had 30 Grade 2 
TABLE 1: Sperm parameters pre- and postoperatively.

\begin{tabular}{|c|c|c|c|c|c|}
\hline $\begin{array}{l}\text { Patient characteristic } \\
\text { Mean }( \pm \text { SD })\end{array}$ & Group 1 & $P^{*}$ & Group 2 & $P^{* *}$ & $P^{* * *}$ \\
\hline Preoperative sperm count $\left(10^{6} / \mathrm{mL}\right)$ & $18.01 \pm 1.88$ & & $17.03 \pm 7.6$ & & 0.356 \\
\hline Postoperative sperm count $\left(10^{6} / \mathrm{mL}\right)$ & $38.40 \pm 1.32$ & 0.03 & $30.1 \pm 4.7$ & 0.04 & 0.04 \\
\hline Preoperative percentage of motile spermatozoa & $17.9 \pm 8.9$ & & $16.3 \pm 6.6$ & & 0.565 \\
\hline Postoperative percentage of motile spermatozoa & $24.7 \pm 89.1$ & 0.001 & $20.6 \pm 5.5$ & 0.03 & 0.03 \\
\hline Preoperative percentage of normal forms & $7.01 \pm 3.02$ & & $5.04 \pm 7.05$ & & 0.285 \\
\hline Postoperative percentage of normal forms & $11.4 \pm 2.1$ & 0.001 & $10.5 \pm 4.21$ & 0.001 & 0.485 \\
\hline
\end{tabular}

$P^{*}$ : comparisons of the preoperative and postoperative values for Group 1.

$P^{* *}$ : comparisons of the preoperative and postoperative values for Group 2.

$P^{* * *}$ : comparisons of postoperative values between groups.

and 26 Grade 3 varicoceles; Group 2 had 24 Grade 2 and 24 Grade 3 varicoceles. The exclusion criteria were having Grade 1 varicocele, previous infertility treatment (antioxidant drug treatment, hormonal treatment), chronic disease, smoking, hormonal abnormality, and/or previous scrotal surgery.All the patients were performed left microsurgical subinguinal spermatic vein ligation with an operation microscope under 19x magnification (Zeiss, Germany).

The mean patient age was $28.6(18-31)$ in Group 1 and 27.8 (19-33) in Group 2. Mean duration of infertility is 13.4 months (12-17) for Group 1 and 14.3 for Group 2 (1317). Sperm parameters of both groups were summarized in Table 1.

Groups were compared by the improvement on sperm parameters and spontaneous pregnancy rates at a mean of 19 $( \pm 4)$ months followup.

2.2. Semen Analysis. In all the patients, semen analysis was performed within $1 \mathrm{~h}$ of collection according to the World Health Organization (WHO) guidelines (1999).

2.3. Statistical Analysis. Statistical analysis was performed using Statistical Package for Social Sciences (SPSS) 20 software for MAC (SPSS Inc., Chicago, IL, United States). All the data are presented as mean (range). The distribution of the data was evaluated by the Kolmogorov-Smirnov test. The comparisons of the groups were performed using the Mann-Whitney $U$ test. $P<0.05$ was considered statistically significant.

\section{Results}

Patients were evaluated by spermiogram and color Doppler USG after three months. No recurrences were reported. Postoperative spermiogram parameters are summarized in Table 1. When sperm parameters were compared postoperatively, the significant improvement in total sperm count, motile sperm count percentage, and normal sperm percentage was reported. The groups were compared to each other and the improvement seemed significantly better in Group 1 . There was no statistically significant improvement difference in normal sperm percentage between groups. Spontaneous pregnancy rate after two years was $45 \%$ in Group 1 and $34 \%$ in Group $2(P<0.05)$.

\section{Discussion}

Metabolic syndrome is a common health problem described with hypertension, increased waist circumference, insulin resistance, and dyslipidemia [1]. It is associated with many urologic diseases, especially andrologic ones. West et al. conducted a study in 2008 and showed a two-fold increase in urolithiasis prevalence in patients with metabolic syndrome [9]. Ekeruo et al. conducted a study and claimed that patients with MetS have stone-forming characteristics such as lower urine $\mathrm{pH}$, gout diathesis, hypocitraturia, and hyperoxaluria [10]. Parallel to these reports, there are several publications about the possible link between MetS and BPH [11-13].

Male infertility and MetS have gained a lot of attention lately. In a multivariate analysis conducted by Bener et al., patients with Type 2 diabetes mellitus and have a BMI $>30$ were found to have a three-fold increased risk of infertility [14]. Also, there are other reports claiming a link between BMI and low sperm motility and ejaculate volume $[15,16]$. Many hypotheses were formed on this matter. Kasturi et al. showed a proinflammatory state caused by dyslipidemia and suggested impairment in sperm parameters. We believe that our patients had a similar condition to have lower sperm parameters in MetS group. That results are parallel to the current literature.

Varicocele makes a venous accumulation in scrotum and increases oxidative stress and deteriorates spermatogenesis by increasing temperature [6]. There are papers claiming that the same mechanism is possible in patients with MetS. Shafik and Olfat performed scrotal dissection to patients with idiopathic infertility and found scrotal lipomatosis in patients with obesity [17]. This finding may be helpful to explain why our patients with MetS did not improve after surgery as expected.

Another situation for patients with high BMI is the unsuccessfully assisted reproduction techniques (ART). Gopalkrishnan et al. and Bungum et al., revealed a higher risk of infertility for patients with a BMI $>30[18,19]$. Our data may support the findings since obesity is a part of MetS.

Hyperglycemia, also a criterion for MetS, was proven to have a role in the production of free radicals, which create DNA damage. DNA fragmentation index, which shows DNA damage, was studied several times for the association with MetS $[15,20]$. DNA fragmentation index can be determined 
by Anilin or Toulidin Blue dying. Our study did not cover DNA fragmentation. This is one of the limitations in our study.

In this study, we aimed to show that MetS might be an independent predictor for sperm parameters. Patients with MetS and varicocele improved after surgery, but not as well as the similar group without MetS. This may help to show that MetS can be a factor for male infertility. To our knowledge, our study is one of the first to show the effect of MetS in patients with varicocele. The retrospective nature of the study and low number of patients are the limitations of our study. Prospective studies with higher cohorts may help to support the data obtained.

\section{Conclusion}

MetS is a health problem for developed countries. Its effects on diseases are a new research topic in modern urology. Infertility is one of the most important diseases MetS might cause. We aimed to show its prognostic effect in patients with varicocele. Our study shows a good correlation between MetS and infertility; however, prospective and higher cohort studies should be conducted to support our findings.

\section{References}

[1] A. V. Chobanian, G. L. Bakris, H. R. Black et al., "The Seventh Report of the Joint National Committee on Prevention, Detection, Evaluation, and Treatment of High Blood Pressure: the JNC 7 report," Journal of the American Medical Association, vol. 289, no. 19, pp. 2560-2572, 2003.

[2] A. O. Kadlec, K. Greco, Z. C. Fridirici, S. T. Hart, T. Vellos, and T. M. Turk, "Metabolic syndrome and urinary stone composition: what factors matter most?" Urology, vol. 80, no. 4, pp. 805-810, 2012.

[3] R. K. Lee, D. Chung, B. Chughtai, A. E. Te, and S. A. Kaplan, "Central obesity as measured by waist circumference is predictive of severity of lower urinary tract symptoms," British Journal of Urology International, vol. 110, no. 4, pp. 540-545, 2012.

[4] J. G. Ryan and J. Gajraj, "Erectile dysfunction and its association with metabolic syndrome and endothelial function among patients with type 2 diabetes mellitus," Journal of Diabetes and its Complications, vol. 26, no. 2, pp. 141-147, 2012.

[5] K. A. Abadilla and A. S. Dobs, "Topical testosterone supplementation for the treatment of male hypogonadism," Drugs, vol. 72, no. 12, pp. 1591-1603, 2012.

[6] C. Mallidis, A. Czerwiec, S. Filippi, J. O’Neill, M. Maggi, and N. McClure, "Spermatogenic and sperm quality differences in an experimental model of metabolic syndrome and hypogonadal hypogonadism," Reproduction, vol. 142, no. 1, pp. 63-71, 2011.

[7] L. Dubin and R. D. Amelar, "Varicocele size and results of varicocelectomy in selected subfertile men with varicocele," Fertility and Sterility, vol. 21, no. 8, pp. 606-609, 1970.

[8] A. V. Hirsh, M. J. Kellett, G. Robertson, and J. P. Pryor, "Doppler flow studies, venography and thermography in the evaluation of varicoceles of fertile and subfertile men," British Journal of Urology, vol. 52, no. 6, pp. 560-565, 1980.
[9] B. West, A. Luke, R. A. Durazo-Arvizu, G. Cao, D. Shoham, and H. Kramer, "Metabolic Syndrome and Self-Reported History of Kidney Stones: The National Health and Nutrition Examination Survey (NHANES III) 1988-1994," American Journal of Kidney Diseases, vol. 51, no. 5, pp. 741-747, 2008.

[10] W. O. Ekeruo, Y. H. Tan, M. D. Young et al., "Metabolic risk factors and the impact of medical therapy on the management of nephrolithiasis in obese patients," Journal of Urology, vol. 172, no. 1, pp. 159-163, 2004.

[11] C. Ozden, O. L. Ozdal, G. Urgancioglu, H. Koyuncu, S. Gokkaya, and A. Memis, "The correlation between metabolic syndrome and prostatic growth in patients with benign prostatichyperplasia," European Urology, vol. 51, no. 1, pp. 199-203, 2007.

[12] C. De Nunzio, W. Aronson, S. J. Freedland, E. Giovannucci, and J. K. Parsons, "The correlation between metabolic syndrome and prostatic diseases," European Urology, vol. 61, no. 3, pp. 560-570, 2012.

[13] A. R. Kristal, K. B. Arnold, J. M. Schenk et al., "Race/ethnicity, obesity, health related behaviors and the risk of symptomatic benign prostatic hyperplasia: results from the prostate cancer prevention trial," Journal of Urology, vol. 177, no. 4, pp. 13951400, 2007.

[14] A. Bener, A. A. Al-Ansari, M. Zirie, and A. O. A. A. Al-Hamaq, "Is male fertility associated with type 2 diabetes mellitus?" International Urology and Nephrology, vol. 41, no. 4, pp. 777784, 2009.

[15] J. E. Chavarro, T. L. Toth, D. L. Wright, J. D. Meeker, and R. Hauser, "Body mass index in relation to semen quality, sperm DNA integrity, and serum reproductive hormone levels among men attending an infertility clinic," Fertility and sterility, vol. 93, no. 7, pp. 2222-2231, 2010.

[16] A. O. Hammoud, N. Wilde, M. Gibson, A. Parks, D. T. Carrell, and A. W. Meikle, "Male obesity and alteration in sperm parameters," Fertility and Sterility, vol. 90, no. 6, pp. 2222$2225,2008$.

[17] A. Shafik and S. Olfat, "Lipectomy in the treatment of scrotal lipomatosis," British Journal of Urology, vol. 53, no. 1, pp. 5561,1981

[18] K. Gopalkrishnan, V. Padwal, P. K. Meherji, J. S. Gokral, R. Shah, and H. S. Juneja, "Poor quality of sperm as it affects repeated early pregnancy loss," Archives of Andrology, vol. 45, no. 2, pp. 111-117, 2000.

[19] M. Bungum, P. Humaidan, M. Spano, K. Jepson, L. Bungum, and A. Giwercman, "The predictive value of sperm chromatin structure assay (SCSA) parameters for the outcome of intrauterine insemination, IVF and ICSI," Human Reproduction, vol. 19, no. 6, pp. 1401-1408, 2004.

[20] H. I. Kort, J. B. Massey, C. W. Elsner et al., "Impact of body mass index values on sperm quantity and quality," Journal of Andrology, vol. 27, no. 3, pp. 450-452, 2006. 